\title{
Rate dependent left bundle branch block: Does it have any effect on the myocardial
} perfusion SPECT

Farzaneh Shariati, Vahid Reza Dabbagh Kakhki, Najes Khatoon Ayati, Ramin Sadeghi

Nuclear Medicine Research Center, Imam Reza Hospital, Faculty of Medicine, Mashhad University of Medical Sciences

[Received 3 XII 2012; Accepted 8 I 2013]

\section{To the editor,}

Dear Sir, the recent issue of Nuclear Medicine Review published an interesting study by Gholamrezanezhad et al. regarding rate dependent left bundle branch block and its effect on myocardial perfusion SPECT [1]. They reported four cases with rate dependent LBBB during exercise stress for myocardial perfusion imaging. Myocardial perfusion SPECT imaging showed no evidence of reversible perfusion defects in three of the patients. They concluded that "maybe it is possible to continue exercise tolerance test (ETT) for those patients undergoing myocardial perfusion scintigraphy and developing rate dependent LBBB".

As the authors themselves mentioned in their study, the number of cases in their case report was not statistically enough to draw any definite conclusion. We would like to report a case with rate dependent LBBB during ETT who developed perfusion defects in the septal wall which disappeared on the Dipyridamole stress imaging.

The case was a 45 year old female patient with history of atypical chest pain since 2 years ago. Her rest ECG was normal. The patient had inconclusive ETT one month before. She was referred to our department for myocardial perfusion SPECT for diagnosis of possible coronary artery disease (CAD). Baseline blood pressure was $130 / 90 \mathrm{mmHg}$ and baseline heart rate was 70 . Five minutes after beginning of ETT (stage II of Bruce protocol and at heart rate of 147), the patient developed LBBB on the ECG monitoring (Figure 1).

Correspondence to: Sadeghi Ramin MD

Nuclear Medicine Research Center, Imam Reza Hospital, Faculty of

Medicine, Mashhad University of Medical Sciences

Tel: +98 (511) 8022729

Fax: +98 (511) 8599359

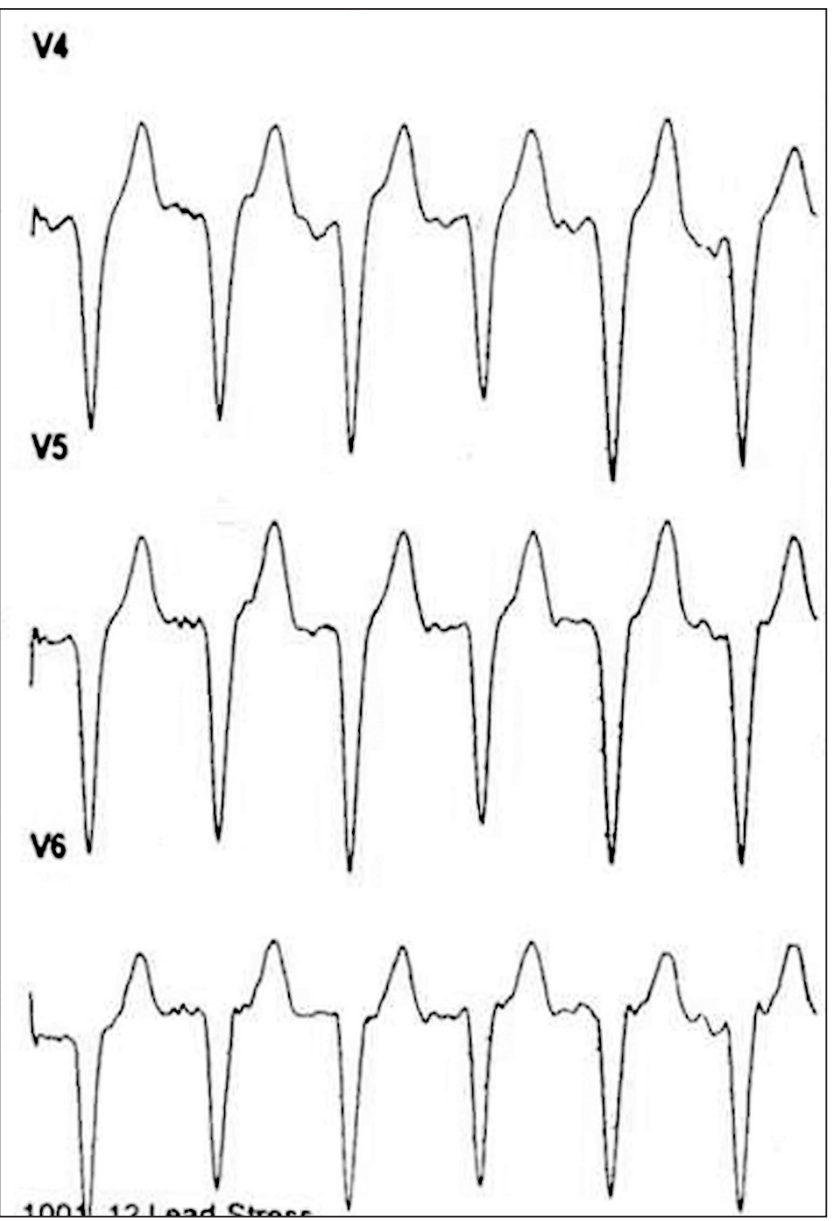

Figure 1. ECG of the patient after development of LBBB during ETT

The ETT was continued till stage III and radiotracer (20 mCi 99mTc MIBI) was injected at heart rate of 160. ETT was continued for 2 minutes post radiotracer injection. During recovery, ECG returned to normal rhythm. Myocardial perfusion SPECT was performed 15 minutes post ETT using a dual head variable angle gamma camera (ECAM Siemens). The scan showed perfusion defects in 


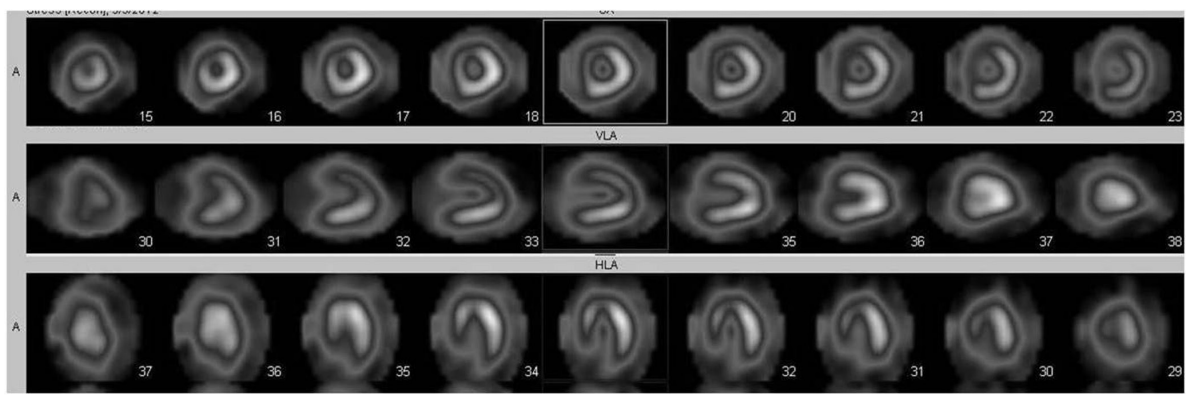

Figure 2. Myocardial perfusion SPECT using ETT stress protocol. Note perfusion defects in the anteroseptal and inferoseptal walls

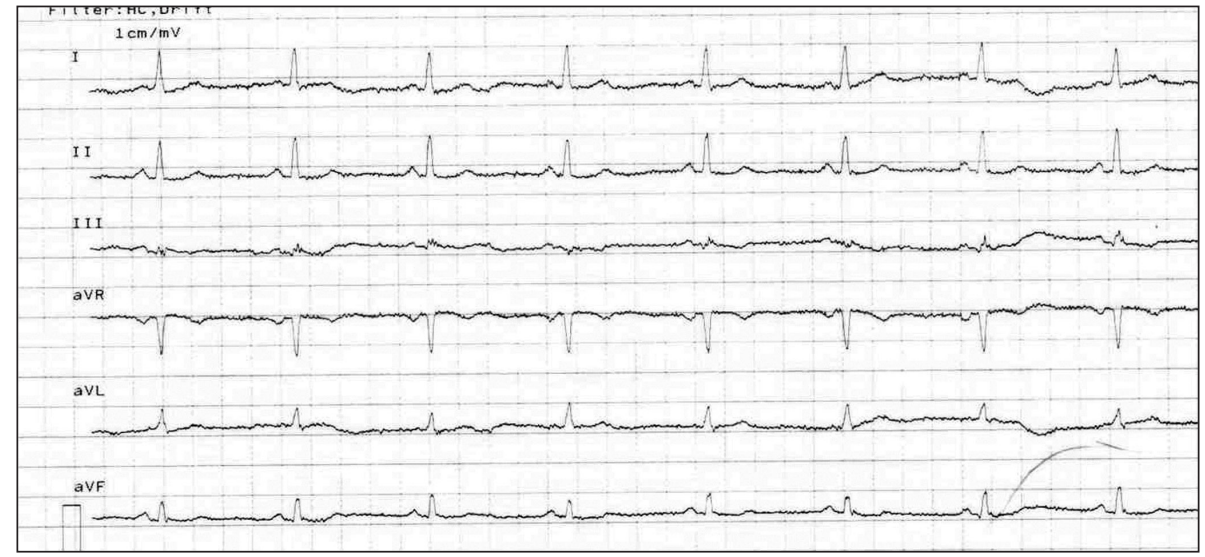

Figure 3. Rest ECG of the patient before pharmacological stress

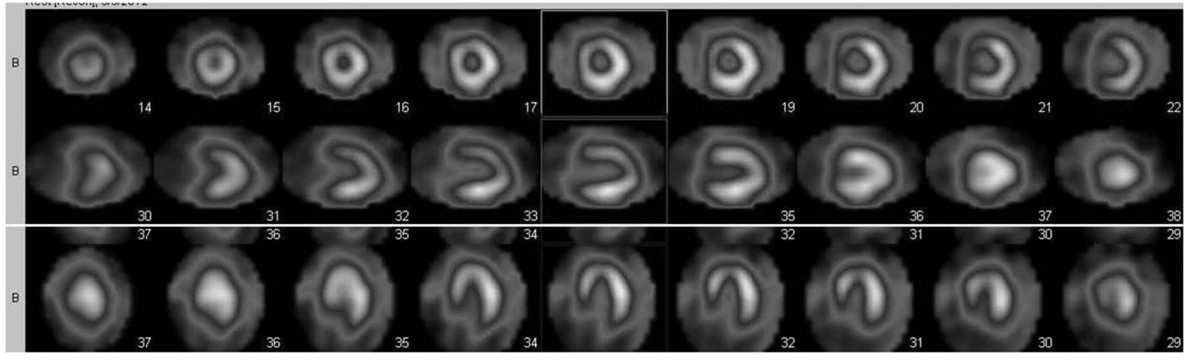

Figure 4. Myocardial perfusion SPECT using pharmacological stress protocol. Note disappearance of perfusion defects in the septal segments

the anteroseptal and inferoseptal walls (Figure 2). The patient was asked to repeat the myocardial perfusion SPECT using Dipyridamole stress protocol. The ECG of the patient was normal (Figure 3) and after slow IV infusion of Dipyridamole, 20 mCi 99m-Tc MIBI was injected intravenously to the patients. 90 minutes post injection myocardial perfusion SPECT was done using the same protocol as the previous imaging [2]. On the pharmacological stress SPECT images the perfusion defects in the anteroseptal and inferoseptal walls disappeared (Figure 4).

Our case shows in contrast to conclusion of Gholamrezanezhad et al., that rate dependent LBBB during ETT can cause important perfusion defects (in the septal wall) on the myocardial perfusion
SPECT images. We recommend repeating myocardial perfusion SPECT imaging using pharmacological stress protocol in these clinical scenarios.

\section{References}

1. Gholamrezanezhad A, Mirpour S, Sarabandi F, Jazayeri B. Rate dependent left bundle branch block: the pattern of myocardial perfusion SPECT. Nucl Med Rev Cent East Eur 2012; 15: 143-148.

2. Sadeghi R, Zakavi SR, Momennezhad M, Dabbagh Kakhki VR. Diffuse Bilateral Breast Uptake on the Myocardial Perfusion SPECT of a Nursing Female. Iran J Nucl Med 2008; 16: 41-44. 